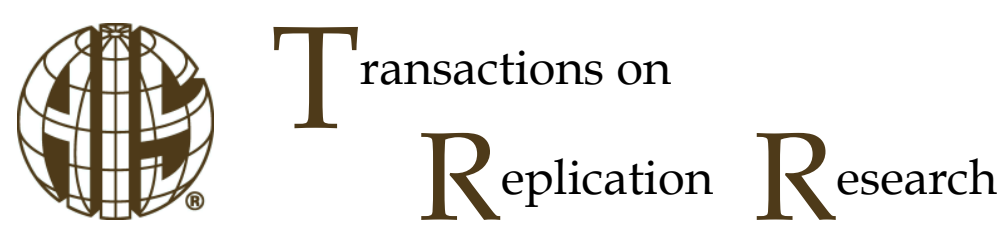

Conceptual Replication

ISSN 2473-3458

\title{
Adaptive System Use Revisited - A Methodological Replication
}

\author{
Phillip Haake \\ University of Mannheim, Institute for Enterprise Systems (InES), Mannheim, Germany \\ Karlsruhe Institute of Technology (KIT), Institute of Information Systems and Marketing (IISM), Karlsruhe, Germany \\ haake@es.uni-mannheim.de / phillip.haake@partner.kit.edu \\ Silvia Schacht \\ Karlsruhe Institute of Technology (KIT), Institute of \\ Information Systems and Marketing (IISM), Karlsruhe, \\ Germany \\ silvia.schacht@kit.edu

\section{Alexander Maedche} \\ Karlsruhe Institute of Technology (KIT), Institute of \\ Information Systems and Marketing (IISM), Karlsruhe, \\ Germany \\ alexander.maedche@kit.edu
}

\section{Abstract:}

"A new concept, adaptive system use (ASU), is conceptualized as a user's revisions of which and how system features are used." (Sun, 2012, p. 453). Sun (2012) conceptualized, tested empirically, and validated this new concept for the first time. We conducted a methodological replication of Sun's (2012) work. We show that his study on ASU can be successfully replicated under similar contextual conditions. While our research is a first step towards understanding ASU in more detail, further replications in the search for new potential moderators and the applicability of ASU in different contextual conditions are necessary. Additional replications further the generalizability of the ASU model and its triggers.

Keywords: Adaptive System Use, ASU, Methodological Replication

The manuscript was received 08/24/2016 and was with the authors 6 months for two revisions.

\section{Introduction}

User adaptation of information systems (IS) is a continuous process in the post-adoption phase. Each interaction of a user with an IS presents the opportunity to revise prevalent use behavior. For instance, an employee might have never used the "track changes"-function in Microsoft (MS) Word and begins to do so, whenever someone shares a document for the purpose of collaborative work on a single document. Sun (2012) motivates his research on user adaptation in post-adoption IS use along these lines. Surprisingly, Sun identified only little research on user adaptation to IS (2012), particularly for the post-adoption stage of IS implementations. Thus, Sun (2012) developed the concept, research model, and measures for Adaptive System Use (ASU) on the basis of Louis and Sutton's (1991) theoretical underpinnings of the understanding of how people switch between automatic and active thinking. His study is focused on a user's revision process for specific features of an IS. An evaluation with 253 office workers largely supported his hypotheses. In this article, we report our methodological replication of his work in a slightly altered context. Thus, we aim to extend the external validity of Sun's (2012) model by replicating it under these adapted circumstances.

We contribute to research with an extension of the body of knowledge on ASU by identifying some indication for its robustness. Before presenting our results, we briefly describe the ASU model as presented by Sun (2012), our applied research methodology, and the data analysis. We conclude the paper with a discussion of our results and the avenues for future research. 


\section{Theoretical Background}

Current conceptualizations of individuals' use of IS are often criticized as simplistic or too lean. As a consequence, research that further opens the black box of use has long been called for and found more attention over the years (e.g. Barki et al. 2007; Burton-Jones and Straub 2006; Elie-Dit-Cosaque and Straub 2011). In particular, ASU has recently been suggested as a concept to capture user adaptation behavior to a new IS (Sun, 2012). Sun (2012) defined the new term of features in use (FIU) as the "basket of system features that are ready to be used by a particular user to accomplish a task" (Sun, 2012, p. 455). ASU involves not only an individual feature of a system, but rather an individual user's FIU. In relation to the aforementioned aspects of FIU, Sun (2012) states that ASU has two sub-dimensions, which are a user's revisions of FIU content and the revisions of FIU spirit, i.e. how the individual uses features. These two dimensions also have sub-dimensions. A revision of the content of FIU, i.e. which features are used by individual users, has the sub-dimensions of "trying new features" (Barki et al., 2007; Jasperson et al., 2005; Sun, 2012) and "feature substituting" (Parthasarathy and Bhattacherjee, 1998; Sun, 2012). Alternatively, users might use features in ways different to those originally assigned by a vendor. This would be a revision of the FIU spirit. The sub-dimensions of the revisions of the spirit of FIU are "feature combining" and "feature repurposing".

Original Model: The original model was developed by Sun (2012) based on Louis and Sutton's (1991) research on the behavioral change of individuals between active and habitual thinking. Louis and Sutton (1991) identified three triggers for active thinking in their original study. These triggers were Novel Situations, Discrepancies, and Deliberate Initiatives. The definition of the triggers is linked to contradictions or interruptions. These contradictions can be, for instance, a misfit between a tool and a task (Kuutti, 1995). A Novel Situation is a situation in which a user has a new task, which requires the use of a new feature or system. This can be linked to the example that an employee needs to work with the "track changes"-function because she started to collaborate on a document with others. A Discrepancy is a contradiction in the current use of a system (Burton-Jones and Straub, 2006). This can be the case if a feature does not create the expected outcomes (Jasperson et al., 2005) because of a contradiction between a feature and a task (e.g., if a user employs vlookup instead of hlookup, whenever only one of the two is appropriate). A Deliberate Initiative is a contradiction between two system use activities. For instance, if a superior asks employees to use a feature that is new to them and the employees have to adapt, learn, and use the new feature.

The aforementioned triggers do not automatically result in a specific adaptation action or active thinking (Louis and Sutton, 1991). It is rather due to an individual's sensemaking of a trigger when a specific action follows. The aforementioned triggers might be present at the same time and influence each other. For instance, if a new employee joins a company and faces novel situations and a discrepancy in the use of technology compared to her work at a previous workplace (Sun, 2012; Louis and Sutton, 1991). Sun (2012) developed a research model on this basic understanding of the adaptation context. Individual and external influencing factors are represented in the overall research model. The individual factors are captured with the measure of personal innovativeness in the use of IT (PIIT). PIIT is defined as an individual trait which is supposed to reflect an individual's willingness to try out new technology (Agarwal and Karahanna, 2000; Agarwal and Prasad, 1999; Sun, 2012). Sun (2012) chose PIIT, because it is a domain specific determinant of individual behavior. External influencing factors are obtained by describing the facilitating conditions comprising the degree to which an individual feels supported by the organizational and technical infrastructure for the use of an IS (Venkatesh et al., 2003, p. 453). The model overall describes just one iteration of ASU. As mentioned before, some triggers and parts of the process may run in parallel, while others run in sequence (Beaudry and Pinsonneault, 2005; Jasperson et al., 2005). The adaptation process continues until the adaptation has closed the discrepancy to a point that the marginal value of another episode of adaptation is too low. Figure 1 depicts Sun's (2012) research model. 


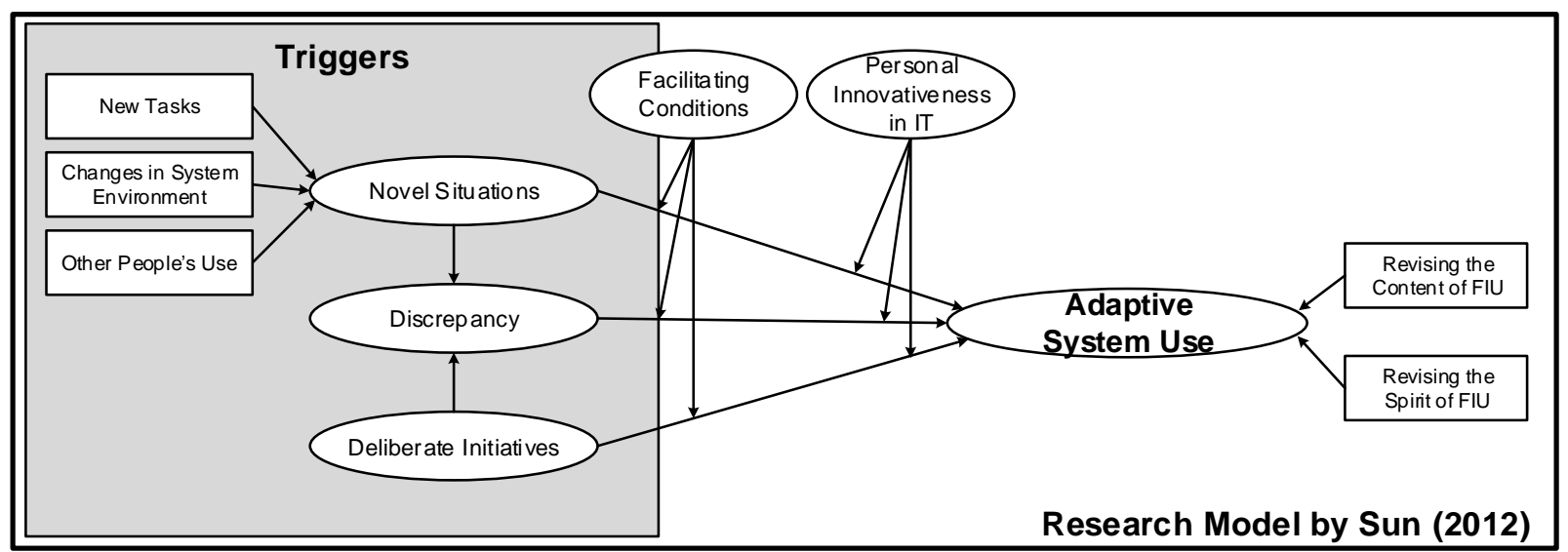

Figure 1. Research Model by Sun (2012)

\section{Research Methodology}

As the main intention of our research is the replication of Sun's (2012) research on ASU in a slightly different context (methodological replication), we applied the research methodology used by Sun (2012). Thus, we also conducted an online survey comprising the items measuring ASU, its triggers, and the moderating factors of personal innovativeness in IT (PIIT) and facilitating conditions (FCOND). Appendix A provides an overview of the measures that we applied in our replication study. However, from a methodological perspective, our research is different in several aspects: First, Sun (2012) conducted the online survey in the US whereas we chose participants from the UK. The national cultures of the UK and the US are similar yet different, therefore, our replication study took place in an altered national culture context (Hofstede, 2011a; 2011b). Second, we focused our investigation explicitly on the use of MS Excel rather than the entire MS Office suite. This allowed us to select panelists who stated that they have a good or very good command of the MS Excel features. We assumed that only these users would have a sufficient system use experience to recall their specific adaptation behavior and educated adaptations. In addition, we also replicated the situating task (Sun, 2012), with a special focus on adaptations of MS Excel. We considered only the participants who reported such an incident in our data analysis.

\subsection{Data Collection}

For our replication study, we collected data by employing a panel service provider to reach a sufficient number of knowledgeable MS Excel users. We are aware that an application of research panels can result in panel effects. In his work, Dennis (2001) examined commonly mentioned panel effects in more detail. These are the creation of "professional respondents" through numerous participations in panel surveys and a selection bias of web-based panels. However, Dennis (2001) noticed that he could "not detect a serious undercurrent of negative panel effects" (Dennis, 2001, p. 36). We ensured the comprehensibility of the items with respect to the language by using respondents from the UK. Before data collection, we provided the panel provider with a set of criteria describing the targeted respondents to reduce the effect of 'professional panelists'. In particular, we focused on potential respondents that can be classified as knowledge workers (Drucker, 1999) and asked the panel provider to balance the set of data with regard to respondents' gender. The overall set of respondents also covers multiple industries and different educational as well as socioeconomic backgrounds to balance the panelists' demographics.

As aforementioned, we decided to collect data from knowledge workers who are experienced in using MS Excel, which we tested using a filter in the survey, asking the participants to assess their perceived experience with MS Excel and to describe an incident they experienced when using MS Excel (see situating task depicted in Appendix B). We decided to consider only the responses of those participants who described a comprehensible incident and who stated that they are very experienced in using MS Excel. This helped us ensure that we did not receive answers from professional respondents, but a rather specific group of people who passed the filter in the survey. Due to the stringent filtering, we had to invite a high number of participants in order to get a sufficient data set. It has been indicated that MS Excel is the most welcomed among identified workarounds (Eckerson and Sherman, 2008) and the toughest system to replace (Robey et al., 2002). Consequently, we selected MS Excel as the information system of interest in our study. Thus, 
our panel is somewhat different to the one used by Sun (2012), as we extend the circle of participants by including knowledge workers in different roles and positions from all kinds of industries into the group of potential survey participants and because we are focusing solely on MS Excel. Furthermore, we conducted the survey with a panel from the UK instead of the US because national culture can also influence the perception and use of IS (Leidner and Kayworth, 2006). Table 1 highlights the differences of our study to the work of Sun (2012).

\begin{tabular}{|l|l|l|}
\hline \multicolumn{3}{|c|}{ Table 1. Characteristics of Panel Study } \\
\hline & Replication Study & Sun (2012) \\
\hline Type of respondents & Knowledge workers from all industries & Employed Administrative staff \\
\hline Respondent residence & UK & USA \\
\hline Researched Information System & Microsoft Excel & Microsoft Office Suite \\
\hline Experience with respective software & high & no information \\
\hline
\end{tabular}

\section{Data Analysis and Results}

For the analysis of our data set, we used the Partial Least Squares (PLS) method as described by Urbach and Ahlemann (2010) and Hair et al. (2013). Sun (2012) also used PLS. We used SmartPLS 3.2.5 to run the PLS for the data analysis to assess the measurement and the structural model.

\subsection{Descriptive Statistics}

After the completion of data collection, we first controlled for the appropriateness of our data by comparing completion times for the surveys and by screening descriptive statistics. Table 2 provides an overview of the descriptive statistics our and Sun's (2012) results.

\begin{tabular}{|l|l|l|}
\hline \multicolumn{3}{|c|}{ Table 2. Descriptive Statistics of Panel Data } \\
\hline & Replication Study & Sun (2012) \\
\hline Number of invitations / participants/ valid data sets & $3,230 / 436 / 281$ & $1,500 / 282 / 253$ \\
\hline Number of female participants & $151(53.7 \%)$ & $180(71 \%)$ \\
\hline Number of male participants & $130(46.3 \%)$ & $73(29 \%)$ \\
\hline Average age & 42.23 years & 37.73 years \\
\hline
\end{tabular}

In total, the panel service provider invited 3,230 panelists to participate in our study. Due to our filter mechanisms, 2,346 participants were screened out, meaning that they either had no or limited experience with MS Excel, could not describe a case of adapting Excel to their needs (situating task depicted in Appendix B), were not employed in an organization, or the gender-related ratio needed to be balanced. From the remaining 884 participants, 111 did not answer at all, and 337 did not answer the questionnaire completely. Thus, we removed them from our overall data set.

Finally, we checked our quality criteria to receive the final data set. We had to remove more than 150 out of 436 completed responses from the panel data set on the grounds of quality issues. For the removal of invalid responses, we followed a two-step approach. First, as we used Questback ${ }^{1}$ as software for online surveys, we were able to calculate a quality measure based on the median response times of all participants. We decided to remove all responses (107 responses) with a quality value smaller than 0.25 , meaning that the participants' response times were $50 \%$ lower than the average of all participants. In a second step, we considered the items that were reversely coded in our survey. Frequently, researchers suggest to use reverse coded items to identify (inter alia insufficient cognitive ability, impaired response accuracy, or actual measurement of a different construct) careless responses (Magazine et al. 1996, p. 247). If respondents answered significantly different on two items that are reversely coded to each other, but referred to the same construct, it can be the case that they were careless in their answers. This implies a lower quality of their

${ }^{1}$ https://www.questback.com/ (Accessed on March 31 $\left.{ }^{\text {st }}, 2017\right)$ 
answers. Again, we removed 48 completed questionnaires based on the analysis of reverse coded items (resulting in 281 valid responses).

\subsection{Measurement Model}

After data collection and cleansing, we validated the measurement model of ASU with respect to its reliability, convergent validity, and discriminant validity. In a first step, we assessed the measurement model's outer loadings. We observed outer loadings between 0.86 and $0.93(>0.708)$ for the reflective constructs of discrepancy (DP) and deliberate initiative (DI). Except for PIIT2 (which was reversely coded; outer loading $=0.312$ ) and FCOND3 (which was also deleted by Sun (2012) in his study), we also had outer loadings between 0.705 and 0.986 for the indicator of our two potential moderators: FCOND and PIIT. Thus, we deleted PIIT2 and FCOND3 to ensure acceptable indicator reliability. As indicated in Table 3, the composite reliabilities (CR) and average variances extracted (AVE) are very similar to Sun's (2012) results. More specifically, the values of CR and AVE exceed the suggested thresholds of 0.7 and respectively 0.5 . Thus, the measurement model fulfills the quality criteria of internal consistency reliability and convergent validity (Hair et al., 2013). The criterion of discriminant validity is also fulfilled, as the AVE's square roots are larger than the correlations among the single constructs (see Table 3).

\begin{tabular}{|c|c|c|c|c|c|c|c|c|c|c|c|c|c|c|c|c|c|c|c|c|}
\hline \multicolumn{21}{|c|}{ Table 3. CR, AVE, and Correlations of First-Order Constructs } \\
\hline \multirow[b]{2}{*}{ Construct } & \multirow{3}{*}{\begin{tabular}{c|}
$\begin{array}{c}\text { No. of } \\
\text { Items }\end{array}$ \\
4
\end{tabular}} & \multirow{2}{*}{\multicolumn{2}{|c|}{ Mean* }} & \multirow{2}{*}{\multicolumn{2}{|c|}{ Std.Dev. }} & \multirow{2}{*}{\multicolumn{2}{|c|}{ CR }} & \multirow{2}{*}{\multicolumn{2}{|c|}{ AVE }} & \multicolumn{11}{|c|}{ Square Roots of AVEs and Correlations** } \\
\hline & & & & & & & & & & 1 & 2 & \begin{tabular}{|l|l}
3 \\
\end{tabular} & 4 & 5 & 6 & 7 & 8 & 9 & 10 & 11 \\
\hline \begin{tabular}{|l|l|} 
1. & ASU (trying new features) \\
\end{tabular} & & 5.59 & 5.58 & 1.54 & 1.45 & .90 & .93 & .70 & .76 & .83 & & & & & & & & & & \\
\hline \begin{tabular}{|l|l}
2. & ASU (feature substituting)
\end{tabular} & 3 & 4.26 & 4.58 & 1.73 & 1.91 & .89 & .91 & .74 & .79 & .36 & .86 & & & & & & & & & \\
\hline ASU (feature combining) & 4 & 4.37 & 4.57 & 1.88 & 1.79 & .89 & .90 & .68 & .71 & .47 & .62 & .82 & & & & & & & & \\
\hline ASU (feature repurposing) & 6 & 3.00 & 3.53 & 1.78 & 1.89 & .95 & .94 & .77 & .73 & .04 & .32 & .36 & .88 & & & & & & & \\
\hline Triggers (new task) & 1 & 4.42 & 3.96 & 1.70 & 1.80 & 1.0 & 1.0 & 1.0 & 1.0 & .26 & .19 & .29 & .08 & 1.0 & & & & & & \\
\hline \begin{tabular}{|l|l}
6. & Triggers (other people's use) \\
\end{tabular} & 3 & 4.14 & 4.16 & 2.03 & 1.94 & .89 & .88 & .73 & .70 & .05 & .23 & .25 & .22 & .12 & .85 & & & & & \\
\hline \begin{tabular}{|l|l}
7. & Triggers (changes in system environment) \\
\end{tabular} & 4 & 3.19 & 3.74 & 1.82 & 1.92 & .86 & .91 & .61 & .72 & .16 & .42 & .38 & .54 & .13 & .37 & .78 & & & & \\
\hline Triggers (discrepancy) & 2 & 3.43 & 4.06 & 1.87 & 1.79 & .93 & .96 & .86 & .92 & .09 & .25 & .19 & .49 & .05 & .30 & .43 & .93 & & & \\
\hline Triggers (deliberate initiative) & 2 & 3.27 & 3.54 & 1.91 & 1.88 & .87 & .89 & .77 & .80 & .01 & .14 & .17 & .34 & .13 & .38 & .41 & .36 & .88 & & \\
\hline \begin{tabular}{|l|l|}
10. & Facilitating conditions \\
\end{tabular} & 2 & 4.73 & 4.61 & 1.64 & 1.78 & .86 & .90 & .76 & .82 & .29 & .15 & .22 & -.05 & .17 & -.11 & -.03 & -.22 & -.09 & .87 & \\
\hline \begin{tabular}{|l|l|}
11. & Personal innovativeness in IT \\
\end{tabular} & 3 & 4.36 & 4.63 & 1.85 & 1.71 & .92 & .92 & .80 & .74 & .30 & .26 & \begin{tabular}{|l|}
.31 \\
\end{tabular} & \begin{tabular}{l|l}
.16 \\
\end{tabular} & .11 & \begin{tabular}{|l|}
.03 \\
\end{tabular} & \begin{tabular}{|l|}
.12 \\
\end{tabular} & \begin{tabular}{|l|}
-.03 \\
\end{tabular} & \begin{tabular}{|l|}
.01 \\
\end{tabular} & \begin{tabular}{|l|}
.40 \\
\end{tabular} & \begin{tabular}{|l|}
.89 \\
\end{tabular} \\
\hline $\begin{array}{l}\text { CR: Composite Reliability } \\
\text { * The mean is the average of the item score } \\
\text { ** The diagonal elements (shaded in grey) a } \\
\text { correlations among constructs } \\
\text { Numbers in grey are the results of Sun's }\end{array}$ & 2) $\mathrm{Wc}$ & k roo & of & & & & & & & & & & & & & & & ement & & \\
\hline
\end{tabular}

\subsection{Structural Model}

Like Sun (2012), we used the latent variable scores for the three formative indicators of the novel situation (NS) construct and the two formative indicators of the ASU construct. In total, we added six single-indicator interaction terms representing the two moderating effects on each of the relationships between the triggers and ASU. These single-indicator interaction terms were directly linked to the dependent variable ASU. After bootstrapping, we were able to assess the relative importance of the formative indicators. The results including the comparison to Sun's (2012) work are summarized in Table 4.

\begin{tabular}{|l|l|l|l|l|l|l|l|}
\hline \multicolumn{8}{|c|}{ Table 4. Variance Inflation Factors and Beta Coefficients of Formative Indicators } \\
\hline & VIF & b-value & \multicolumn{1}{l|}{ t-value } & \multicolumn{1}{l|}{ p-value } \\
\hline Revising content of FIU & 1.139 & 0.402 & 0.59 & 2.631 & 5.68 & 0.009 & $<0.001$ \\
\hline Revising spirit of FIU & 1.139 & 0.770 & 0.52 & 5.916 & 4.75 & $<0.001$ & $<0.001$ \\
\hline New tasks & 1.023 & 0.159 & 0.65 & 1.669 & 4.39 & 0.096 & $<0.001$ \\
\hline Changes in system environment & 1.165 & 0.870 & 0.57 & 15.425 & 3.89 & $<0.001$ & $<0.001$ \\
\hline & 1.161 & 0.189 & -0.11 & 2.150 & 0.56 & 0.032 & NA \\
\hline $\begin{array}{l}\text { Others use } \\
\text { Numbers available in grey represent Sun's (2012) results }\end{array}$ \\
\hline
\end{tabular}

All in all, we verified Sun's (2012) results with slight differences. In contrast to Sun (2012), our data indicate a significant contribution of others' use (OU) to form a novel situation. Thereby, we followed Sun's call to replicate the analysis of OU's effect on novel situation. We could not identify an effect of new tasks (NT) on novel situation. Thus, current results on the formative indicators of novel situation are inconclusive and 
require further analysis. As all VIF values are smaller than 3.3, there are no issues with multicollinearity (Diamantopoulos and Winkelhofer, 2001) of ASU and NS.

As highlighted in Table 5, our data confirm Sun's results (2012) with regard to the direct effects of the three triggers on ASU. Similar to Sun (2012), we could not identify a direct effect of deliberate initiatives (DI) on ASU. We could not support any of Sun's hypotheses regarding the moderating effects, when we added the aforementioned moderators. In other words, we could not observe any interaction or multi-group moderation effects of PIIT and FCOND. These results are also confirmed by the small effect size that results when the moderators are included in the model.

In addition, we conducted a Sobel test (Hayes, 2013) to verify the mediation effect of discrepancy (DP). First, we tested the mediating effect of DP on the relationship between NS and ASU. In the absence of DP, we identified a significant total effect of NS on ASU (see Table 5). Introducing DP as mediator, the direct influence of NS on ASU remains significant. As the 95 percent confidence interval $(\mathrm{Cl}$ of 0.327 to 0.423$)$ does not contain zero, we can conclude that DP has a partial mediation effect on the relationship between NS and ASU. Second, we identified a full mediation effect of DP on the relationship between DI and ASU, as there is a non-significant relationship between DI and ASU (see Table 5) and the confidence interval $(\mathrm{Cl}$ of 0.269 to 0.370 ) also does not contain zero. Like Sun (2012), we conducted a cluster analysis to identify heterogeneous triggering conditions and to examine behavioral patterns of ASU under these conditions. Since our results are similar to Sun's (2012), we present these findings in Appendix C.

\begin{tabular}{|c|c|c|c|c|}
\hline \multicolumn{5}{|c|}{ Table 5. Results of Structural Model } \\
\hline & \multicolumn{2}{|c|}{ Effects Only } & \multicolumn{2}{|c|}{ Direct Effects + Moderating Effect } \\
\hline \multicolumn{5}{|l|}{ a. Dependent Variable: ASU } \\
\hline$R^{2}$ & 0.46 & 0.47 & 0.48 & 0.50 \\
\hline$\Delta R^{2}$ & & & $0.02\left(f^{2}=0.04\right)$ & $0.03\left(f^{2}=0.06\right)$ \\
\hline Novel Situation (NS) & $0.46^{* * *}$ & $0.23^{* *}$ & $0.47^{* * *+1}$ & $0.24^{* *}$ \\
\hline Discrepancy (DP) & $0.27^{\star * *}$ & $0.31^{* *}$ & $0.27^{* * *}$ & $0.26^{* *}$ \\
\hline Deliberative Initiative (DI) & -0.01 (n.s.) & 0.04 (n.s.) & -0.03 (n.s.) & 0.08 (n.s.) \\
\hline $\begin{array}{l}\text { Facilitating Conditions } \\
\text { (FCOND) }\end{array}$ & 0.09 (n.s.) & $0.28^{* *}$ & 0.10 (n.s.) & $0.30^{* *}$ \\
\hline Personal Innovativeness in IT & $0.23^{*+*+}$ & $0.23^{* *}$ & $0.23^{* \star \star}$ & $0.24^{* *}$ \\
\hline $\mathrm{NS} \times \mathrm{FCOND}$ & & & -0.04 (n.s.) & 0.09 (n.s.) \\
\hline $\mathrm{DP} \times \mathrm{FCOND}$ & & & -0.05 (n.s.) & -0.13 (n.s.) \\
\hline $\mathrm{DI} \times \mathrm{FCOND}$ & & & 0.01 (n.s.) & -0.03 (n.s.) \\
\hline NS $\times$ PIIT & & & -0.06 (n.s.) & $0.14^{*}$ \\
\hline DP $\times$ PIIT & & & -0.07 (n.s.) & -0.03 (n.s.) \\
\hline $\mathrm{DI} \times \mathrm{PIIT}$ & & & 0.06 (n.s.) & $-0.14^{*}$ \\
\hline \multicolumn{5}{|c|}{ b. Dependent Variable: Discrepancy } \\
\hline $\mathrm{R}^{2}$ & 0.23 & 0.32 & 0.22 & 0.32 \\
\hline Novel Situation (NS) & $0.35^{* * *}$ & $0.41^{* *}$ & $0.35^{\text {t*t+ }}$ & $0.41^{* *}$ \\
\hline Deliberative Initiative (DI) & $0.20^{* * *+}$ & $0.23^{*+2}$ & $0.20^{* * *}$ & $0.23^{* *}$ \\
\hline
\end{tabular}

\section{Discussion}

The present study replicates the work of Sun (2012), who conceptualized ASU and developed measures for the related constructs and triggers of ASU. Generally, our data confirm his research results in our slightly altered context (see also Table 6). Our replication demonstrates the applicability of Sun's (2012) items for the three triggers of NS, DI, and DP as well as ASU. Our data set supports all relationships of the triggers to ASU and the mediating effect of DP. However, we have different results with regard to the impact of OU and NT based on our data. While Sun (2012) indicated a significant effect of NT and a non-significant effect of OU on NS, we observed opposite results.

We assume that the variations in results can be related to the slight differences between the contexts of the two studies. In a context where several tools can be applied (such as the use of the whole MS Office suite (Sun, 2012)), new tasks could have a significant effect on the perception of novel situations because 
different solutions can be employed to deal with the new tasks. They might not have a particular effect when looking at the use of a specific software solution such as MS Excel. Spreadsheet software has been designed for the solution of rather specific kinds of problems, which share similar characteristics. Furthermore, we selected a panel of proficient users of MS Excel, who have the ability to make use of the features of the software and might be expert users in their organization. It could be the case that because of their level of specialization these knowledge workers experience a much smaller number of new tasks than general administrative staff members, who were the respondents in Sun's (2012) study. However, OU can be important for the user types that are included in our survey because expert users are likely to benefit from an exchange of best practices with colleagues. These kinds of benefits of exchange between users might not be so important in purely administrative settings as covered by Sun's (2012) research, were many clerks or office assistants might tend to work on their own.

\begin{tabular}{|l|l|l|}
\hline \multicolumn{2}{|c|}{ Table 6. Summary of Hypotheses Testing } & Sun (2012) \\
\hline Hypotheses & Supported? & Y \\
\hline H1: Novel Situations $\rightarrow$ ASU & Y & Y \\
\hline H2: Discrepancies $\rightarrow$ ASU & Y & N \\
\hline H3: Deliberate initiatives $\rightarrow$ ASU & $\mathrm{N}$ & Y \\
\hline H4: Novel situations $\rightarrow$ Discrepancies & $\mathrm{Y}$ & N \\
\hline H5: Deliberate initiatives $\rightarrow$ Discrepancies & Y & \\
\hline $\begin{array}{l}\text { H6: Facilitating Conditions moderate the impact of (6a) novel } \\
\text { situations, (6b) discrepancies, and (6c) deliberate initiatives, } \\
\text { on ASU. }\end{array}$ & $\mathrm{N}$ & $\begin{array}{l}\text { Partially. PIIT did not } \\
\text { moderate the relation- } \\
\text { ship between discre- } \\
\text { p7: Personal Innovativeness of IT use positively moderates } \\
\text { the impact of (7a) novel situations and (7b) discrepancies, and } \\
\text { negatively moderates the impact of (7c) deliberate initiatives, } \\
\text { on ASU. }\end{array}$ \\
\hline
\end{tabular}

In addition, we could not support any hypotheses with regard to the moderators presented by Sun (2012). We tested for the moderating effect of PIIT and FCOND because we wanted to explore whether the theoretically well-grounded argument for the moderating effect of these two factors would hold in a slightly altered setting (see Table 5). It became evident that this was not the case. Sun (2012) identified a positive moderation of PIIT for the relationship between NS and ASU as well as a negative moderation of PIIT for the relation between DI and ASU. However, even those two moderating effects could not be identified based on our data set. Thus, while he identified little support for the hypotheses, we found even less. This might be related to the effect size of the moderating effects, which tend to be rather small. Future research could avoid this issue with an even larger sample, which potentially increases statistical power and therefore the ability to detect a rather small moderating effect.

We assume that the lack of an identifiable moderating effect of PIIT and FCOND can also be due to the characteristics of MS Excel and the experience of the user types, which were the respondents for our replication study. The explanation for the lack of a positive moderating effect for the relationship of NS and ASU can also be linked to the work environment of the respondents. As aforementioned, the respondents in our panel are likely to be users that are more specialized because they perceived themselves as experienced users of MS Excel. This might mean that they had fewer opportunities to perceive their own user behavior to be particularly innovative, since they were much more familiar with the technology at their disposal. They have fewer opportunities to experiment and explore something new, as the items for PIIT suggest it: Hence, not innovativeness but knowledge and the kind of learning approach of individuals is likely to be a more appropriate moderator for this relationship. Authors of future replication studies should therefore consider including constructs of system knowledge and individual learning behavior as potential moderators. Furthermore, Bala and Venkatesh (2013) state that the initial response to change in process characteristics or work in general will be influenced by the characteristics of the underlying technology. Thus, it could be the case that technology characteristics moderate the relationship between the triggers of novel situations, deliberate initiatives, and perceived discrepancies and the state of ASU (Haake et al., 2015).

A negative moderation of the relationship between DI and ASU by PIIT as identified by Sun (2012) would mean a resistance to change because of demands of others (Sun, 2012). The absence of such a moderating 
effect in our study can also be explained with the likely nature of the predominant work environment of our panelists compared to those used by Sun (2012). Expert users are likely to work in more autonomous functions then general administrative staff, which is much more likely to be in a situation where a larger number of people can judge the execution of a job. Furthermore, very detailed processes and protocols for the execution of tasks might exist. These conditions are probably not the usual work environment of our panelists, who are likely to conduct specialized tasks, which require their specific knowledge of MS Excel. It could also be the case that these differences in the observations are rooted in different working cultures of the UK and the US. As we cannot provide conclusive results for the differences between our and Sun's (2012) observations, more replications of the ASU model in varying contexts and with differing software artifacts are warranted as only our slight adaptation already produced significantly different results. Additional replications would further increase the generalizability of the ASU model and its triggers.

As we replicated Sun's work (2012), our research has similar limitations. A first limitation stems from the application of a panel for data collection. However, as discussed previously, we invested substantial effort in mitigating issues related to the application of a panel service provider. Another limitation stems from our decision for MS Excel as the system of interest, since the system allows a great degree of freedom for the end user. Regarding this, our study does not differ significantly from Sun's (2012) original study. Further research on software artifacts, which have more restrictions for adaptation (e.g. ERP, CRM systems) would address this current research gap. In addition, our study was conducted at one single point of time. Similar to Sun (2012), we suggest the analysis of multiple feedback loops in future longitudinal studies.

\section{Conclusion}

ASU can be replicated successfully under similar conditions. Our research is a first step towards understanding ASU in more detail. Further replications to identify other potential moderators and to reassert the applicability of ASU in different contexts are necessary. 


\section{References}

Agarwal, R., \& Karahanna, E. (2000). Time flies when you're having fun: Cognitive absorption and beliefs about information technology usage. MIS Quarterly, 24(4), 665-694.

Agarwal, R., \& Prasad, J. (1999). Are individual differences germane to the acceptance of new information technologies? Decision Sciences, 30(2), 361-391.

Bala, H., \& Venkatesh, V. (2013). Changes in employees job characteristics during an enterprise system implementation: A latent growth modeling perspective. MIS Quarterly, 37(4), 1113-1140.

Barki, H., Titah, R., \& Boffo, C. (2007). Information system use - related activity: An expanded behavioral conceptualization of individual-level information system use. Information Systems Research, 18(2), 173-192.

Beaudry, A., \& Pinsonneault, A. (2005). "Understanding user responses to information technology: A coping model of user adaptation," MIS Quarterly, 29(3), 493-524.

Burton-Jones, A., \& Straub, D. W. (2006). "Reconceptualizing system usage: An approach and empirical test", Information Systems Research, 17(3), 228-246.

Dennis, J. M. 2001. Are internet panels creating professional respondents? Marketing Research, 13(2), 3438.

Diamantopoulos, A., \& Winkelhofer, A. H. (2001). Index construction with formative indicators: An alternative to scale development. Journal of Marketing Research, 38(2), 269-277.

Drucker, P. F. (1999) Knowledge-worker productivity: The biggest challenge. California Management Review, 41(2), 79-94.

Eckerson, W., \& Sherman, R. P. (2008). Strategies for managing spreadmarts. Business Intelligence Journal 13(1), 23-24.

Elie-Dit-Cosaque, C. M., \& Straub, D. W. (2011). Opening the black box of system usage: User adaptation to disruptive IT. European Journal of Information Systems, 20(5), 589-607.

Hair, J. F. J., Hult, G. T. M., Ringle, C., \& Sarstedt, M. (2013). A primer on partial least squares structural equation modeling (PLS-SEM). Los Angeles, CA: Sage Publications.

Hayes, A. F. 2013. Introduction to mediation, moderation, and conditional process analysis: A regressionbased approach: Methodology in social sciences, New York, NY: Guildford Publications.

Hofstede, G. 2011a. Dimensionalizing cultures: The Hofstede model in context. Online Readings in Psychology and Culture, 2(1), 1-26.

Hofstede, G. 2011b. (2016, August 4). Country comparison. Itim International. Retrieved from: https://geerthofstede.com/united-kingdom.html.

Jasperson, J. S., Carter, P. E., \& Zmud, R. W. (2005). A comprehensive conceptualization of post-adoptive behaviors associated with information technology enabled work systems. MIS Quarterly, 29(3), 525557.

Kuutti, K. (1995). Activity theory as a potential framework for human- computer interaction research. In B. A. Nardi (Ed.), Context and consciousness: Activity theory and human-computer interaction (pp. 1744). Cambridge, MA: MIT Press.

Leidner D. E., \& Kayworth T. (2006). A review of culture in information systems research: Toward a theory of information technology culture conflict. MIS Quarterly, 30(2), 357-399.

Louis, M. R., \& Sutton, R. I. (1991). Switching cognitive gears: From habits of mind to active thinking. Human Relations, 44(1), 55-76.

Magazine, S. L., Williams, L. J., \& Williams, M. L. (1996). A confirmatory factor analysis examination of reverse coding effects in Meyer and Allen's affective and continuance commitment scales. Educational and Psychological Measurement, 56(2), 241-250. 
Parthasarathy, M., \& Bhattacherjee, A. (1998). Understanding post-adoption behavior in the context of online services. Information Systems Research, 9(4), 362-379.

Robey, D., Ross, J. W., \& Boudreau, M. C. (2002). Learning to implement enterprise systems: An exploratory study of the dialectics of change. Journal of Management Information Systems, 19(1), 17-46.

Sun, H. (2012). Understanding user revisions when using information system features: Adaptive system use and triggers. MIS Quarterly, 36(2), 453-478.

Urbach, N., \& Ahlemann, F. (2010). Structural equation modeling in information systems research using partial least squares. Journal of Information Technology Theory and Application, 11(2), 5-40.

Venkatesh, V., Morris, M. G., Davis, G. B., \& Davis, F. D. (2003). User acceptance of information technology: Toward a unified view. MIS Quarterly, 27(3), 425-478. 


\section{Appendix A: Questionnaire}

\section{Personal Innovativeness (PIIT)}

(adapted from Agarwal and Karahanna 2000)

Please indicate to what extent you agree with the following statements about that incident you reported. (7Point Likert scale from $1=$ strongly disagree to $7=$ strongly agree)

PIIT1: If I heard about a new information technology, I would look for ways to experiment with it.

PIIT2: In general, I am hesitant to try out new information technology (reverse coded; dropped).

PIIT3: $\quad$ Among my peers, I am usually the first to try out new information technologies.

PIIT4: I like to experiment with new information technologies.

\section{Facilitating Conditions (FCOND)}

(adapted from Venkatesh et al. 2003)

During that incident reported above... (7-Point Likert scale from $1=$ strongly disagree to $7=$ strongly agree)

FCOND1: I had the resources necessary to change.

FCOND2: I had the knowledge necessary to change.

FCOND3: A specific person (or group) was available for assistance for that change (dropped).

\section{Triggers}

(adapted from Sun 2012)

Please indicate to what extent you agree with the following statements about that incident you reported. (7Point Likert scale from $1=$ strongly disagree to $7=$ strongly agree)

New task (NT):

NT1: $\quad$ My task changed (e.g., I had a new task).

Changes in system environments (SE):

SE1: $\quad$ The system environment of Excel in my organization changed.

SE2: $\quad$ Our Excel was being upgraded.

SE3: $\quad$ The peripheral facilities (e.g., printers, copiers, and scanners) changed in my organization.

SE4: I used different versions of Excel.

Other people's use (OU):

OU1: I saw other people's use of that feature.

OU2: $\quad$ Someone showed me a new feature.

OU3: $\quad$ Someone showed me a new way of using a feature I knew.

Discrepancy $(D P)$ :

DP1: $\quad$ Some Excel features did not work as I thought.

DP2: $\quad$ There were discrepancies between what I expected and what I found out in terms of the features in Excel. 
Deliberate initiative (DI):

DI1: $\quad$ Somebody asked me to use certain features.

DI2: I was forced by others to change.

\section{Adaptive System Use (ASU)}

(adapted from Sun 2012)

Please indicate to what extent you agree with the following statements about that incident you reported. (7-

Point Likert scale from 1 = strongly disagree to 7 = strongly agree)

Trying new features (TR):

TR1: I played around with features in Excel.

TR2: $\quad$ I used some Excel features by trial and error.

TR3: I tried new features in Excel.

TR4: $\quad$ I figured out how to use certain Excel features.

Feature substituting (FS):

FS1: I substituted Excel features that I used before.

FS2: I replaced some Excel features with new features.

FS3: I used similar Excel features in place of the features at hand.

Feature combining $(F C)$ :

FC1: $\quad$ I generated ideas about combining features in Excel that I was using.

FC2: I combined certain features in Excel.

FC3: I used some features in Excel together for the first time.

FC4: I combined features in Excel with features in other applications to finish a task.

Feature repurposing (FR):

FR1: I applied some features in Excel to tasks that the features are not meant for.

FR2: $\quad$ I used some features in Excel in ways that are not intended by the developer.

FR3: $\quad$ The developers of Excel would probably disagree with how I used some features in Excel.

FR4: $\quad$ My use of some features in Excel was likely at odds with its original intent.

FR5: $\quad$ I invented new ways of using some features in Excel.

FR6: I created workarounds to overcome Excel's restrictions. 


\section{Appendix B: The Situating Task}

Situating Task

In this survey, we define features as the building blocks of a software package. You know them as functions such as the "copy", "paste", "Sort", and "PivotTable" functions in Microsoft Excel. First, please recall one incident or situation in which you changed your use of some features in Microsoft Excel for your work. By changes in using features, we mean you change your feature selection in Microsoft Excel or you change the way you use Microsoft Excel features. For instance, you tried new features, you combined some features for the first time, or applied features to tasks that they are not meant for, etc.

Please use several sentences to describe what happened during that incident. For example, why did you change the use of the feature? What did you do? How did you learn to do that? (An answer to this question is required.)

\section{Appendix C: Cluster Analysis}

Similar to the work of Sun (2012), we conducted a two-step approach to perform the cluster analysis. The cluster analysis serves to classify cases of the overall study (in this study 281 cases) into groups being as homogenous as possible within each group, but as heterogeneous as possible among the different groups. In the first step, we performed a hierarchical cluster analysis using SPSS (version 23) to identify the number of clusters. Like Sun (2012), we used the Ward's minimum variance method for cluster formation and Euclidean distances as the similarity measure. Finally, a three-cluster solution seemed to result in meaningful pattern. In the second step, we conducted a $K$-means cluster analysis where we defined the $K$ value to be three. The ANOVA indicated significant differences among the three identified clusters (see Table C1). Based on the cluster analysis, we were able to analyze the degree of differentiation of each cluster to the other ones based on the triggers. Table $\mathrm{C} 2$ summarizes the results by presenting the means, standard deviations and the differentiation tests.

\begin{tabular}{|c|c|c|c|c|c|}
\hline \multicolumn{6}{|c|}{ Table C1. ANOVA Results of the Three Clusters } \\
\hline & $\begin{array}{l}\text { Mean Square } \\
\text { Cluster }\end{array}$ & \multicolumn{2}{|c|}{\begin{tabular}{l|l} 
Mean Square \\
Error
\end{tabular}} & $\mathbf{F}$ & Significance \\
\hline Novel Situation & 42.419 & \multicolumn{2}{|l|}{0.908} & 46.738 & .000 \\
\hline Discrepancies & 284.693 & \multicolumn{2}{|l|}{0.982} & 289.86 & .000 \\
\hline Deliberate Initiative & 204.356 & 1.193 & & $171.27 \varepsilon$ & .000 \\
\hline \multicolumn{6}{|c|}{ Table C2. Cluster Center and Comparison } \\
\hline & \multicolumn{4}{|c|}{$\begin{array}{l}\text { Mean (S.D.) of Cluster Groups and Patterns of } \\
\text { Triggers }\end{array}$} & \multirow{2}{*}{$\begin{array}{l}\text { Significant Contrast Values } \\
\text { (Bonferroni tests) }\end{array}$} \\
\hline & $\begin{array}{l}\text { Cluster } 1 \\
(n=146)\end{array}$ & $\begin{array}{l}\text { Cluster } 2 \\
(n=59)\end{array}$ & \multicolumn{2}{|c|}{$\begin{array}{l}\text { Cluster } 3 \\
(\mathrm{n}=76)\end{array}$} & \\
\hline Novel Situation & $\begin{array}{l}4.28(0.84) \\
\text { high }\end{array}$ & $\begin{array}{l}4.19(1.02) \\
\text { high }\end{array}$ & \multicolumn{2}{|c|}{$\begin{array}{l}3.02(1.09) \\
\text { low }\end{array}$} & $1-2^{* * *} ; 1-3^{* * *} ; 2-3$ (n.s.) \\
\hline Discrepancies & $\begin{array}{l}4.79(0.97) \\
\text { high }\end{array}$ & $\begin{array}{l}2.10(0.82) \\
\text { low }\end{array}$ & \multicolumn{2}{|c|}{$\begin{array}{l}1.83(1.13) \\
\text { low }\end{array}$} & $1-2$ (n.s.); $1-3^{* * *} ; 2-3^{* * *}$ \\
\hline Deliberate Initiative & $\begin{array}{l}3.92(1.25) \\
\text { high }\end{array}$ & $\begin{array}{l}4.22(1.19) \\
\text { high }\end{array}$ & \multicolumn{2}{|c|}{$\begin{array}{l}1.30(0.56) \\
\text { low }\end{array}$} & $1-2$ (n.s.); $1-3^{* * *} ; 2-3^{* * *}$ \\
\hline
\end{tabular}


In two clusters, we came to the same results as Sun (2012). We also identified one cluster, which can be referred to as intensive triggering. This cluster is characterized by high levels of all three ASU triggers and contains (as in Sun's work) the most cases $(n=146)$. Another cluster - also identified by Sun $(2012)-$ is the cluster of non-intensive triggering characterized by low levels of all triggers (cluster 3 ). Only, the characteristics of cluster 2 differ from Sun's (2012) results. While Sun identified a cluster, he named discrepancy triggering and which has only a high level of the trigger discrepancy, we identified a cluster having high levels of novel situation and deliberate initiative. Due to the differing results, we conducted a two-step clustering analysis as provided by SPSS (version 23), which combines the two steps conducted by Sun (2012) (meaning combination of hierarchical and k-means clustering approach). The two-step clustering resulted in two categories, namely the intensive triggering conditions and the non-intensive triggering conditions (see Table C3).

\begin{tabular}{|c|c|c|c|}
\hline \multicolumn{4}{|c|}{ Table C3. Cluster Center and Comparison } \\
\hline & \multicolumn{2}{|c|}{ Mean (S.D.) of Cluster Groups and Patterns of Triggers } & \multirow{2}{*}{$t$ values } \\
\hline & Cluster $1(n=151)$ & Cluster $2(n=130)$ & \\
\hline Novel Situation & $4.47(0.73)$ - high & $3.28(1.11)-$ low & $10.79^{* * *}$ \\
\hline Discrepancies & 4.45 (1.21) - high & $2.23(1.48)-$ low & $13.87^{* * *}$ \\
\hline Deliberate Initiative & 4.31 (1.03) - high & 2.07 (1.34) - low & $15.79^{* * *}$ \\
\hline \multicolumn{4}{|l|}{$* * 0<0.001$} \\
\hline
\end{tabular}

As stated by Sun "a potentially thorny but essential issue in cluster analysis is the selection of the number of clusters" (2012, p. A8). Thus, the existence of a third cluster remains questionable. Therefore, our remaining cluster analysis refers to the clusters of high and non-triggering conditions. Based on the two identified clusters, we performed in the next step independent samples t-tests to test differences in ASU. The analysis revealed that - similar to Sun's (2012) results - the means of trying new features did not significantly differ between the groups. Thus, this first-order sub-construct of ASU seems to have no impact on the characteristics of the two clusters (see Table C4).

\begin{tabular}{|c|c|c|c|}
\hline \multicolumn{4}{|c|}{ Table C4. Adaptive System Use in Different Triggering Conditions } \\
\hline & Mean (S.D.) of Cluste & & \\
\hline & Cluster $1(n=151)$ & Cluster $2(n=130)$ & t values \\
\hline Trying new features & $5.69(1.10)$ & $5.47(1.43)$ & 1.42 (n.s.) \\
\hline Feature substituting & $4.61(1.13)$ & $3.86(1.73)$ & $4.32^{* * *}$ \\
\hline Feature combining & $4.76(1.17)$ & $3.92(1.77)$ & $4.77^{* * *}$ \\
\hline Feature repurposing & $3.67(1.40)$ & $2.23(1.37)$ & $8.72^{*+*+}$ \\
\hline
\end{tabular}




\section{About the Authors}

Phillip Haake. Since October 2015, he is an associated Ph.D. student in the research group of professor Maedche at the Karlsruhe Institute of Technology (KIT). He completed his dissertation in September 2017, which he had started at the University of Mannheim in December 2013. While working on his dissertation about understanding the effective use of information systems, he also worked as a consultant at a local omnichannel retailer and in a project by the initiative Usability-in-Germany, which was founded by the German federal ministry for economic affairs and energy. Prior to this, he had earned his M.Sc. in the Mannheim Master in Management program as well as his B.Sc. in business administration at the University of Mannheim.

Silvia Schacht is post-doctoral research assistant in the research group of professor Maedche at the Karlsruhe Institute of Technology (KIT) since November 2015. She had already had this role at the University of Mannheim, since January 2015. Silvia Schacht had been a Ph.D. student at the chair of professor Maedche from August 2009 until December of 2014, where she wrote a dissertation on preventing organizations to lose the knowledge acquired in projects. She has received several best paper wards for her work (DESRIST Conference 2013, 2015, 2016) and the HMD Best Paper Award 2016. Silvia Schacht studied business informatics at the University of Mannheim from 2003 until 2009. Prior to her studies, she worked as a chemical laboratory assistant at BASF AG in Ludwigshafen from 1999 until 2003.

Alexander Maedche is full professor for information systems and service design at the Karlsruhe Institute of Technology (KIT) since November 2015. From 2009 until 2015, he was full professor and chair of information systems at the University of Mannheim. He studied business engineering at the Karlsruhe Institute of Technology from 1994 until 1999. Alexander Maedche stayed at the Karlsruhe Institute of Technology to pursue a Ph.D. from 1999 until 2001. After finishing his Ph.D., he became the department manager of a research group at the Forschungszentrum Informatik in Karlsruhe from 2001 until 2003. Subsequently, he became the department manager for business intelligence at Bosch Group from 2003 until 2006 and the Vice President for product management and user interaction at SAP AG from 2006 until 2009 before returning to academia.

Copyright (C) 2018 by the Association for Information Systems. Permission to make digital or hard copies of all or part of this work for personal or classroom use is granted without fee provided that copies are not made or distributed for profit or commercial advantage and that copies bear this notice and full citation on the first page. Copyright for components of this work owned by others than the Association for Information Systems must be honored. Abstracting with credit is permitted. To copy otherwise, to republish, to post on servers, or to redistribute to lists requires prior specific permission and/or fee. Request permission to publish from: AIS Administrative Office, P.O. Box 2712 Atlanta, GA, 30301-2712 Attn: Reprints or via e-mail from ais@aisnet.org. 